\title{
ANDES

\section{Síndrome de ACNES, explorando la pared abdominal como noxa del dolor abdominal}

\author{
ACNES syndrome, exploring the abdominal wall as source of abdominal pain
}

\author{
Clara García R. ${ }^{\text {, }}$ Gonzalo Botija A. ${ }^{\mathrm{a}}$, Aránzazu Recio L. ${ }^{\mathrm{a}}$, Consuelo Nieto I. ${ }^{\mathrm{b}}$, Alfonso Barrio M. ${ }^{\mathrm{a}}$
}

aDepartamento de Gastroenterología, Hepatología y Nutrición Pediátrica, Hospital Universitario Fundación Alcorcón. Madrid. España.

bUnidad del Dolor, Hospital Universitario Fundación Alcorcón. Madrid. España.

Recibido: 18 de enero de 2021; Aceptado: 20 de septiembre de 2021

¿Qué se sabe del tema que trata este estudio?

El síndrome de ACNES (Anterior Cutaneous Nerve Entrapment Syndrome) es un tipo de dolor abdominal originado en la pared abdominal cuyo diagnóstico se basa en la historia clínica y exploración física compatibles.

\begin{abstract}
¿Qué aporta este estudio a lo ya conocido?
Se presenta un estudio descriptivo en menores de 17 años con el objetivo de dar a conocer el ACNES en este rango de edad. Para ello hemos descrito una serie de 20 pacientes detallando sus características demográficas y clínicas que permitan su diagnóstico, así como el tratamiento inicial administrado.
\end{abstract}

\section{Resumen}

El dolor de pared abdominal, y concretamente el síndrome de ACNES (Anterior Cutaneous Nerve Entrapment Syndrome), forma parte del diagnóstico diferencial del dolor abdominal crónico. Este síndrome es poco conocido y por consiguiente infradiagnosticado. Objetivos: Describir las características clínicas y evolutivas de pacientes diagnosticados de ACNES y llamar la atención sobre esta patología. Pacientes y Método: Estudio descriptivo retrospectivo realizado en un centro de referencia, entre octubre 2016 y julio de 2021, en pacientes menores 17 años, diagnosticados de ACNES, que cumpliesen al menos dos de cuatro hallazgos siguientes: signo de Carnett, Pinch test, disestesias en el punto de máximo dolor, mejoría tras infiltración de anestésico local, habiéndose descartado patología abdominal visceral o funcional. Se recogieron variables epidemiológicas, síntomas, exploración física, pruebas complementarias, tratamiento y evolución. Se usaron estadísticas descriptivas. Resultados: 20 pacientes diagnosticados de ACNES, 75\% mujeres, mediana de edad 12,85 años. Se objetivó signo de Carnett en 95\%, signo de Pinch test en 65\% y disestesias en 90\% de los pacientes. 65\% refirió síntomas pseudoviscerales. 7 pacientes presentaron sobrepeso u obesidad. La localización más frecuente (50\%) fue la fosa ilíaca derecha, a nivel T10-T11. Un paciente refirió mejoría espontánea; 7 mejoraron con analgesia oral; 9 fueron derivados a la Unidad del Dolor, de los cuales acudieron 5, los que mejoraron con infiltración anestésica con bupivacaína-triamcinolona; los 4 restantes perdieron
Palabras clave: Dolor Abdominal; Síndrome de Atrapamiento del Nervio Cutáneo Anterior; Dolor de Pared Abdominal; Dolor Abdominal Crónico; Signo de Carnett
Correspondencia:

Clara García R.

claragr2391@gmail.com
Editado por:

Luisa Schonhaut Berman 
seguimiento. Conclusión: El ACNES debe ser considerado en pacientes con dolor abdominal crónico. La combinación de hallazgos típicos en la historia y el examen físico permiten su diagnóstico, evitando pruebas complementarias innecesarias. El tratamiento debe ser escalonado, comenzando por analgesia oral, seguido de infiltración anestésica y, por último, neurectomía anterior.

\section{Keywords:}

Abdominal Pain;

Anterior Cutaneous

Nerve Entrapment

Syndrome;

Abdominal Wall Pain;

Chronic Abdominal

Pain;

Carnett's Sign

\section{Introducción}

El dolor abdominal crónico (DAC) presenta una alta prevalencia (10-19\%) en las consultas de Atención Primaria y de Atención Especializada ${ }^{1,2}$. Según su etiología se clasifica en: visceral, cuando se origina en órganos intraabdominales; parietal, originado en la pared abdominal; y funcional, definido por los criterios ROMA IV y basado en la alteración del eje intestinocerebro $^{3-5}$.

Un tipo de DAC parietal es el síndrome de ACNES (Anterior Cutaneous Nerve Entrapment Syndrome), descrito por primera vez en 1926 por J. Carnett ${ }^{6}$. Cada nervio cutáneo anterior atraviesa un canal fibroso neurovascular localizado cerca del borde lateral del músculo recto anterior del abdomen, hasta llegar a la piel. El dolor aparece mediante compresión y/o tracción de los nervios o bien, cuando se produce fibrosis ${ }^{3,7-9}$. Un estudio reciente sobre el ACNES a nivel histológico, descarta la posibilidad de infección o inflamación como causa del mismo ${ }^{10}$.

En el síndrome de ACNES el dolor se localiza en la unión del tercio externo y medio del músculo recto anterior del abdomen, más frecuentemente a nivel de dermatomas T10 y T11 siendo el 86\% unilateral y el 13-14\% bilateral. El cuadrante más afectado es el inferior derecho (55-75\%), lo que supone descartar una apendicitis aguda con la consiguiente realización de exploraciones complementarias e incluso ingresos hospitalarios $^{5,9,11-12}$.

Clínicamente el cuadro se caracteriza por un dolor selectivo, localizado en un área pequeña (del tamaño de la huella dactilar del médico). Empeora con el juego, tos, ejercicio y los cambios posturales ${ }^{7,9,13}$. El inicio es gradual en la mayoría de los pacientes, pero según un estudio, el 50\% de los pacientes presenta un inicio brusco y lancinante ${ }^{7}$. Un estudio de 139 pacientes lo clasifica como intenso en la escala EVA (escala visual analógica $)^{9}$.

En cuanto a los hallazgos en la exploración física, indicaremos al paciente que se coloque en decúbito supino. A continuación, se localizará a punta de dedo el área de máximo dolor, en la unión del tercio medio y tercio externo del músculo recto abdominal anterior, zona de emergencia de la rama del nervio cutáneo anterior. Una vez localizado, se procede a comprimir dicho punto, y sin soltar, se solicita al paciente que contraiga la musculatura abdominal ("mírate el ombligo"). Si dicha maniobra aumenta notablemente el dolor, se refiere como signo de Carnett positivo (figu- 
ra 1). Esta maniobra lo diferencia del dolor de origen visceral, pues en este caso, se alivia el dolor. De igual modo, el pinzamiento y elevación del panículo adiposo suprayacente al punto de máximo dolor, produce un aumento desproporcionado del dolor en comparación con el lado contralateral sano, ya que el nervio atrapado no se desliza por el túnel fibroadiposo por el que atraviesa el músculo; conocido como signo de Pinch test positivo (figura 2). Por último, la demostración de la alteración de la sensibilidad cutánea (disestesias), para el tacto y la temperatura, del área lesionada mediante una torunda impregnada en alcohol, en comparación con el lado contralateral, apoya fuertemente el diagnóstico $3,5,6,9,14-15$.

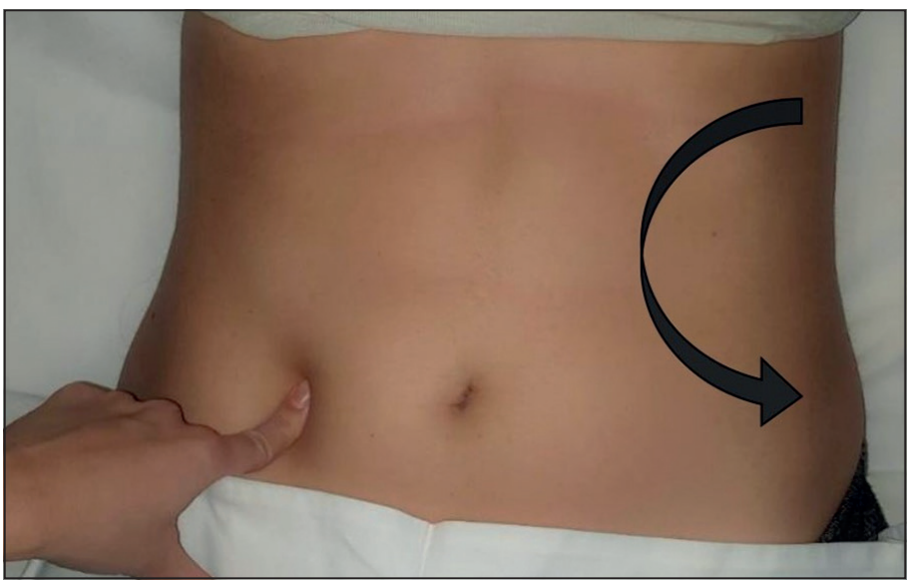

Figura 1. Signo de Carnett: la compresión del punto de máximo dolor y simultáneamente la contracción de la musculatura abdominal ("mírate el ombligo"), produce empeoramiento del dolor.

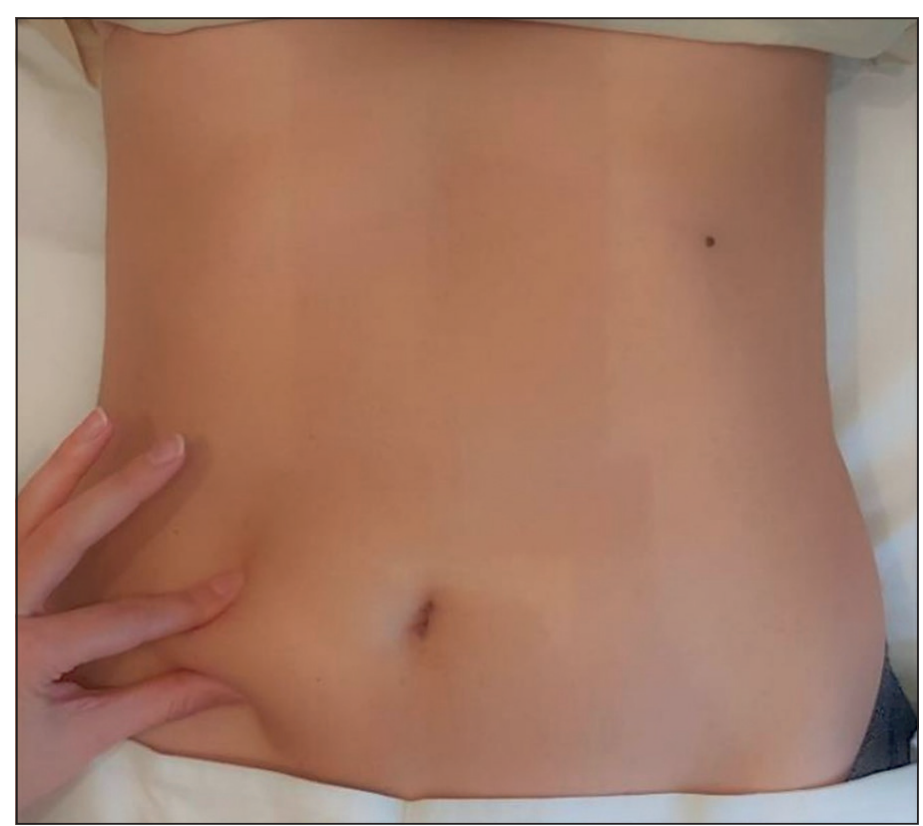

Figura 2. Pinch test: el pinzamiento y elevación del panículo adiposo suprayacente al punto de máximo dolor, produce un aumento desproporcionado del dolor en comparación con el lado contralateral sano.
Con frecuencia se acompaña de síntomas 'pseudoviscerales' por alteración del sistema nervioso autónomo incluyendo náuseas, distensión abdominal, pérdida de apetito, disminución del peso o alteración del ritmo intestinal ${ }^{11,16-17}$.

Al igual que en la evaluación de cualquier dolor abdominal, se deben descartar síntomas de alarma que sugieran una patología orgánica, como fiebre, pérdida de peso involuntaria, disfagia, vómitos persistentes, cambio repentino del ritmo intestinal, hematemesis, melenas, hematoquecia y antecedentes familiares de cáncer o enfermedades gastrointestinales (como la enfermedad inflamatoria intestinal o enfermedad celíaca) ${ }^{13}$.

Uno de los diagnósticos diferenciales más frecuentes es el síndrome de intestino irritable, para lo cual recientemente se ha publicado un cuestionario pediátrico validado de 17 ítems, con una sensibilidad del $85 \%$ y especificidad del $89 \%{ }^{18}$. Otros diagnósticos diferenciales se muestran en la tabla $1^{7,19-23}$.

Respecto del tratamiento, ha de ser secuencial basado en la gravedad y la duración de los síntomas. Inicialmente se puede comenzar con analgésicos y modificaciones del estilo de vida. En caso de no mejoría, el tratamiento de elección es la infiltración de anestésico local en el punto de máximo dolor ${ }^{7,9,24}$ y si persiste el dolor tras infiltraciones repetidas se puede valorar tratamiento quirúrgico mediante neurectomía anterior $^{7,9}$.

Revisiones recientes del DAC, llaman la atención sobre la falta de conocimiento del dolor abdominal de origen parietal, centrándose exclusivamente en el dolor abdominal visceral y funcional, suponiendo, en algunos casos, un exceso de pruebas complementarias innecesarias o invasivas, así como el diagnóstico erróneo y el retraso del mismo ${ }^{3,25,26}$.

Los objetivos de este estudio son describir las características clínicas y evolutivas de pacientes diagnosticados de ACNES y llamar la atención sobre esta patología, escasamente conocida.

\section{Pacientes y Método}

Estudio descriptivo retrospectivo de pacientes menores de 17 años seleccionados de la consulta de Gastroenterología Pediátrica y hospitalización entre octubre 2016 y julio de 2021, del Hospital Universitario Fundación Alcorcón, Comunidad de Madrid (España). Se trata de un hospital secundario, perteneciente al sistema público de salud, que atiende a una población aproximada de 171.098 habitantes, de los cuales 29.000 son menores de 17 años. El hospital dispone de todas las subespecialidades Pediátricas, excepto Cirugía Pediátrica. 
Se identificaron los pacientes a través de la historia clínica electrónica. Se empleó para su búsqueda todos aquellos diagnósticos que incluyesen la palabra "ACNES" en pacientes menores de 17 años, ya fuesen procedentes de consulta o del informe de alta hospitalaria. Dicho diagnóstico fue realizado por los gastroenterólogos pediátricos del hospital.

Se seleccionaron aquellos pacientes que presentaran una historia clínica y exploración física compatibles con diagnóstico de ACNES y cumpliesen al menos dos de los cuatro hallazgos siguientes: signo de Carnett, Pinch test, disestesias en el punto de máximo dolor o mejoría tras infiltración de anestésico local ${ }^{12}$.

Según la intensidad del dolor el médico decide el tratamiento a seguir: 1) analgesia oral y modificación de actividad física, en los síntomas leves; 2) infiltración con anestésico local (bupivacaína-triamcinilona en nuestro centro) en los casos con dolor moderadosevero o que no mejoraron tras analgesia oral tras 2-4 semanas de tratamiento ${ }^{12}$. Si el paciente no mejora en los primeros 6 meses de seguimiento se deriva a hospital terciario (con Cirugía Pediátrica) para valorar neurectomía anterior.

Se excluyeron aquellos pacientes en los que se confirmó una patología abdominal visceral o funcional, como causa final de los síntomas a través de anamnesis, exploraciones físicas seriadas y pruebas complementarias. Las pruebas complementarias empleadas fueron: analítica sanguínea (hemograma, bioquímica, función renal, hepática y tiroidea, proteína $\mathrm{C}$ reactiva), ecografía abdominal, gastroscopia y parásitos en heces ${ }^{7,13}$.

Se definió sobrepeso a aquellos pacientes que presentaran un IMC entre los percentiles 85-97 y obesidad, percentil mayor de 97, según las gráficas del Estudio Transversal Español de crecimiento 2010.

El análisis estadístico se realizó mediante "IBM SPSS Statistics", versión 17 presentándose frecuencias absolutas y relativas para variables cualitativas, mediana y rango intercuartílico en el caso de variables cuantitativas.

Se analizaron variables epidemiológicas, antropométricas, exploratorias (localización, signo de Carnett, Pinch test, disestesias) y síntomas acompañantes. Se describieron las pruebas complementarias realizadas y el tratamiento administrado.

El protocolo de estudio fue revisado y aprobado por el Comité de Ética del Hospital.

\section{Resultados}

Durante el periodo de estudio se diagnosticaron 21 pacientes de síndrome de ACNES. Se excluyó un paciente por rechazo del consentimiento informado. De los 20 pacientes restantes, el $75 \%$ fueron mujeres, con
Tabla 1. Diagnósticos diferenciales ACNES y entidades que imitan ACNES $8,20-24$

Síndrome de dolor miofascial abdominal

Hernia (de Spiegel, umbilical, epigástrica)

Hematoma

Lordosis lumbar exagerada / diferencia de longitud de pierna

Hernia de deportista o ingle / pubalgia deportiva

Neurofibroma/quistes subaracnoideos, Schwannoma

Radiculopatía

Desgarro de la pared abdominal

Anomalías en las costillas

Síndrome de costilla deslizante

Tumor de la pared abdominal (desmoides)

Endometriosis de la pared abdominal

Anormalidades de la columna vertebral

Hernia de disco

Atrapamiento del nervio ilioinguinal o iliohipogástrico

Radiculopatía diabética

Infección por herpes

Tejido cicatricial (apendicectomía, cesárea, laparoscopia)

ACNES: Anterior Cutaneous Nerve Entrapment Syndrome.

una mediana de edad de 12,85 años (IQ 11,18-14,10). Cinco de los pacientes presentaron sobrepeso (IMC p85-97) y 2 pacientes obesidad (IMC mayor al p97). El $75 \%$ de los pacientes habían nacido en España. El 30\% (6/20) de los pacientes, 4 varones, tenía antecedentes psiquiátricos, entre los que destacan depresión, TDAH y trastorno adaptativo (tabla 2).

Previo al diagnóstico, 12 pacientes (60\%) habían consultado en Urgencias por DAC y precisaron ingreso para control del dolor y estudio 5 pacientes (25\%). La mayoría de los pacientes $80 \%$ (16/20), fueron diagnosticados en consulta ambulatoria. La mediana de tiempo desde el inicio del dolor hasta consultar en Atención Especializada fue de 2 meses (IQ 1-4) y el tiempo desde el inicio del dolor hasta el diagnóstico fue de 2 meses (IQ 2-6,25).

En la exploración física se objetivó signo de Carnett positivo en el 95\% (19/20), Pinch test en el 65\% $(13 / 20)$ y disestesias en el $90 \%$ (18/20) de los pacientes. Presentaron síntomas pseudoviscerales el 65\% (13/20) de los pacientes, siendo los más frecuentes los vómitos $20 \%(4 / 20)$ y la diarrea $40 \%(8 / 20)$. Un paciente refirió pérdida de peso. La localización más frecuente del dolor fue la fosa ilíaca derecha a nivel de T10-11 (50\%), fosa ilíaca izquierda (40\%) y bilateral (10\%) (tabla 3). 
Tabla 2. Características demográficas de los pacientes con ACNES

\begin{tabular}{ll}
\hline Variable de interés & Resultados \\
\hline Sexo (hombre:mujer) & $1: 3(5 / 15,75 \%$ mujeres) \\
Edad (mediana) & 12,85 años (IQ 11,18-14,10) \\
Nacionalidad española & $75 \%$ \\
Antecedentes psiquiátricos & $30 \%(6 / 20)$ \\
Antropometría: & \\
$\quad$ Índice de masa corporal (mediana) & $19,09($ IQ 17,31-26,23) \\
$\quad$ Sobrepeso (IMC p85-97) & $5 / 20(25 \%)$ \\
$\quad$ Obesidad (IMC mayor p97) & $2 / 20(10 \%)$ \\
\hline
\end{tabular}

IMC: índice de masa corporal; ACNES: Anterior Cutaneous Nerve Entrapment Syndrome.

\begin{tabular}{ll}
$\begin{array}{l}\text { Tabla 3. Características del dolor } \mathbf{y} \text { exploración física de pacientes } \\
\text { con ACNES }(\mathbf{n}=\mathbf{2 0})\end{array}$ \\
\hline Variable de interés & Resultados \\
\hline Signo Carnett & $95 \%(19 / 20)$ \\
Pinch test & $65 \%(13 / 20)$ \\
Alteración sensibilidad (disestesias) & $90 \%(18 / 20)$ \\
Localización (dermatomas T10-11) & \\
Bilateral & $10 \%(2 / 20)$ \\
Unilateral & $90 \%(18 / 20)$ \\
Derecha & $50 \%(10 / 20)$ \\
Izquierda & $40 \%(8 / 20)$ \\
Síntomas pseudoviscerales & $65 \%(13 / 20)$ \\
\hline ACNES: Anterior Cutaneous Nerve Entrapment Syndrome.
\end{tabular}

Las pruebas complementarias solicitadas previas al diagnóstico de ACNES fueron: analítica sanguínea $80 \%(16 / 20)$, ecografía $70 \%(14 / 20)$, gastroscopía $20 \%$ (4/20) y parásitos en heces $20 \%(4 / 20)$.

Se pautó tratamiento con antiinflamatorios y analgésicos orales en todos los pacientes, desapareciendo la sintomatología en 7 pacientes (35\%). Un paciente refirió mejoría espontánea y se perdió el seguimiento de 3 pacientes. Se derivó a la Unidad del Dolor para infiltración con bupivacaína-triamcinolona a 9 pacientes $(45 \%)$. Un paciente rechazó el tratamiento y otros 3 no acudieron a la Unidad del Dolor. Tras la primera infiltración desapareció el dolor en 3 pacientes de los 5 tratados. De los dos restantes, uno precisó una segunda infiltración y otro se derivó a un hospital terciario con Cirugía Pediátrica para valoración de neurectomía anterior por persistencia de dolor tras 2 infiltraciones. Dicha intervención no se realizó por desaparición del dolor tras una nueva infiltración guiada por ecografía en el hospital terciario.

\section{Discusión}

El dolor abdominal originado en la pared del abdomen y concretamente el ACNES, debe formar parte del diagnóstico diferencial del dolor abdominal crónico. No obstante, actualmente su exploración sistemática continúa siendo ignorada ${ }^{3,25,26}$, probablemente por el "pensamiento visceral" de los clínicos a la hora de evaluar al paciente con dolor abdominal, como refiere Bishop $^{26}$. El objetivo de este estudio es dar a conocer este síndrome. Para ello hemos descrito una serie de 20 pacientes detallando sus características demográficas y clínicas que permitan su diagnóstico, así como el tratamiento inicial administrado.

Pese a este desconocimiento, en las series descritas recientemente por Siawash M et al refieren una prevalencia en población pediátrica de ACNES del 13\% de los pacientes diagnosticados inicialmente como dolor abdominal funcional ${ }^{11}$. Al igual que nuestra muestra, presenta una clara predisposición por el sexo femenino $(75 \%)$ y una edad media alrededor de los 15 años ${ }^{3,5}$.

Se han descrito factores de riesgo en adultos que predisponen esta situación, destacando obesidad, cicatrices quirúrgicas abdominales, embarazo, ascitis y sobreuso de la musculatura abdominal ${ }^{3,12}$. No obstante, hasta en un $60 \%$ de los casos no se observan factores de riesgo que lo precipiten ${ }^{27}$. La prevalencia de sobrepeso y obesidad de los pacientes con ACNES (35\%), es muy similar a la descrita en la población española (30\%) según un reciente estudio de $2020^{28}$.

El diagnóstico es eminentemente clínico, basándose en la historia clínica y exploración física, presencia de signos de Carnett, Pincht test y disestesias en el $95 \%, 65 \%$ y $90 \%$ de nuestros pacientes respectivamente, lo cual concuerda con la bibliografía previamente publicada $^{5,9,12}$.

El $65 \%$ presentaron síntomas pseudoviscerales, siendo el más frecuente la diarrea. Se ha postulado la aparición de estos síntomas por la relación entre los nervios intercostales afectos y los órganos internos por cadenas nerviosas esplácnicas. Estos síntomas son muy inespecíficos y pueden retrasar el diagnóstico ${ }^{3,7,11}$.

Nuestros pacientes presentan una prevalencia del $30 \%$ de trastornos de salud mental infanto-juvenil que, aunque es mínimamente superior a la población general española $(20 \%)^{29}$, consideramos que se debe tener en cuenta como factor de riesgo.

En relación al tratamiento, inicialmente es fundamental informar a los pacientes y sus familias de la benignidad del proceso. En Pediatría ha de ser secuencial, de tal forma que aquellos pacientes con síntomas leves pueden mejorar con analgesia oral (antiinflamatorios o paracetamol) y cambios en las actividades diarias, como ocurrió en el 35\% de nuestros pacientes. Se de- 
ben evitar actividades que impliquen ejercicios vigorosos de musculatura abdominal, siendo útil el uso de fajas abdominales, calor local, masajes y rehabilitación ${ }^{13}$.

En aquellos pacientes con síntomas moderados o severos (el dolor interfiere con sus actividades cotidianas) o no se experimenta mejoría con analgesia oral, está indicada la infiltración de anestésico local en el punto de máximo dolor con o sin corticoides, guiado o no por ecografía, con una respuesta exitosa (reducción de la escala EVA superior al 50\%) entre el 38 y el $87 \%$ de los pacientes ${ }^{7,9,24}$. Un estudio descartó el efecto placebo al demostrar ausencia de mejoría con infiltración de suero salino ${ }^{30}$.

La infiltración ecoguiada puede ser más costosa, pero ofrece mayor precisión en la infiltración y menor riesgo de inyectar en cavidad peritoneal ${ }^{13}$.

La asociación de anestésico local y corticoides (principalmente lidocaína-betametasona) probablemente sea más eficaz que el uso de anestésicos exclusivamente, aunque no existe consenso en este sentido ${ }^{31}$.

En la mayoría de los estudios realizados en población adulta, la confirmación diagnóstica se realiza mediante la mejoría clínica tras la infiltración de anestésico local en el área lesionada ${ }^{7,9,12}$, no obstante estudios pediátricos no lo precisan para su diagnóstico definitivo $^{13}$.

El 45\% de nuestros pacientes fueron derivados a la Unidad del Dolor, y de ellos, todos los que aceptaron el tratamiento con infiltraciones presentaron mejoría o desaparición del dolor. En aquellos pacientes con dolor severo y que no responden a las infiltraciones repetidas durante más de 6 meses, puede estar indicada la realización de neurectomía anterior con una tasa de éxito a corto plazo del $78-85 \%$ y escasos efectos secundarios ${ }^{7,9,32-33}$.

La terapia con radiofrecuencia pulsada ha sido recientemente demostrada como una alternativa eficaz y mínimamente invasiva a considerar en pacientes en los que ha fallado el tratamiento conservador, previo a la neurectomía, siendo esta última más eficaz, pero con mayor número de complicaciones potenciales (hematoma, infección) y la necesidad de anestesia general $^{34}$.

Nuestro estudio presenta limitaciones, como son el limitado número de pacientes incluidos en el estudio, la variabilidad inter-observador, la falta de adherencia al tratamiento con infiltración del nervio por ser una técnica invasiva y la falta de seguimiento a largo plazo de los pacientes que permita conocer posibles recaídas.

El síndrome de ACNES debe ser considerado en niños con dolor abdominal crónico, principalmente mujeres adolescentes, con historia clínica compatible y exploración física característica. El diagnóstico es clínico, mediante la positividad de los signos de Carnett, Pinch test y disestesias en el área de máximo dolor, evitando pruebas complementarias innecesarias.

\section{Responsabilidades Éticas}

Protección de personas y animales: Los autores declaran que los procedimientos seguidos se conformaron a las normas éticas del comité de experimentación humana responsable y de acuerdo con la Asociación Médica Mundial y la Declaración de Helsinki.

Confidencialidad de los datos: Los autores declaran que han seguido los protocolos de su centro de trabajo sobre la publicación de datos de pacientes.

Derecho a la privacidad y consentimiento informado: Los autores han obtenido el consentimiento informado de los pacientes y/o sujetos referidos en el artículo. Este documento obra en poder del autor de correspondencia.

\section{Conflicto de intereses}

Los autores declaran no tener conflicto de intereses.

\section{Agradecimientos}

Elia Pérez de la Unidad de Investigación del Hospital Universitario Fundación Alcorcón, quien gentilmente brindó asistencia en el análisis estadístico y metodológico. Al equipo de médicos de la Unidad de Gastroenterología Pediátrica del Hospital Universitario Fundación Alcorcón, por su colaboración en el desarrollo y redacción de este artículo. A las familias que participan en el estudio.

\section{Referencias}

1. Chitkara DK, Rawat DJ, Talley NJ. The epidemiology of childhood recurrent abdominal pain in western countries: a systematic review. Am J Gastroenterol. 2005;100 (8):1868-75.
2. Korterink J, Devanarayana NM, Rajindrajith S, et al. Childhood functional abdominal pain: mechanisms and management. Nat Rev Gastroenterol Hepatol. 2015;12(3): 159-71.

3. Akhnikh S, de Korte N, de Winter P. Anterior cutaneous nerve entrapment syndrome (ACNES): the forgotten diagnosis. Eur J Pediatr. 2014;173(4):4459.

4. Hyams JS, Di Lorenzo C, Saps M, et al. Functional Disorders: Children and Adolescents. Gastroenterology 2016; 150:1456-68. 
5. Siawash M, Roumen R, Ten WTA, et al. Diagnostic characteristics of anterior cutaneous nerve entrapment syndrome in childhood. Eur J Pediatr. 2018;177(6):835-9.

6. Carnett J. Intercostal neuralgia as a cause of abdominal pain and tenderness. Surg Gynecol Obstet. 1926;42:8

7. Scheltinga MR, Roumen RM. Anterior cutaneous nerve entrapment syndrome (ACNES). Hernia 2018;22(3):507-16.

8. Applegate WV, Buckwalter NR. Microanatomy of the structures contributing to abdominal cutaneous nerve entrapment syndrome. J Am Board Fam Pract. 1997;10(5):329-32.

9. Boelens OB, Scheltinga MR, Houterman $S$, et al. Management of anterior cutaneous nerve entrapment syndrome in a cohort of 139 patients. Ann Surg. 2011;254(6):1054-8.

10. Markus J, van Montfoort M, de Jong JR, et al. Histopathologic examination of resected nerves from children with anterior cutaneous nerve entrapment syndrome: ¿Clues for pathogenesis? J Pediatr Surg. 2020;55(12):2783-6.

11. Siawash M, de Jager-Kievit JW, Ten WT, et al. Prevalence of Anterior Cutaneous Nerve Entrapment Syndrome in a Pediatric Population With Chronic Abdominal Pain. J Pediatr Gastroenterol Nutr. 2016;62(3):399-402.

12. Mol FMU, Maatman RC, De Joode LEGH, et al. Characteristics of 1116 Consecutive Patients Diagnosed With Anterior Cutaneous Nerve Entrapment Syndrome (ACNES). Ann Surg. 2021;273(2):373-8

13. Kamboj AK, Hoversten P, Oxentenko AS. Chronic Abdominal Wall Pain: A Common Yet Overlooked Etiology of Chronic Abdominal Pain. Mayo Clin Proc 2019;94(1):139-44.

14. Carnett JBBW. The treatment of intercostal neuralgia of the abdominal wall. Ann Surg 1933; 98:820-9.

15. Van Assen T, Brouns JA, Scheltinga MR, et al. Incidence of abdominal pain due to the anterior cutaneous nerve entrapment syndrome in an emergency department. Scand J Trauma Resusc Emerg Med. 2015;23:19.

16. Davis JH. Segmental neuralgia in childhood simulating visceral disease. JAMA. 1936 107:1620-6.

17. Hughes GS Jr, Treadwell EL, Miller J. Syndrome of the rectus abdominis muscle mimicking the acute abdomen. Ann Emerg Med. 1985;14(7):694-5.

18. Siawash M, van Assen T, Tjon A Ten W, et al. Abdominal Wall Pain or Irritable Bowel Syndrome: Validation of a Pediatric Questionnaire. J Pediatr Gastroenterol Nutr. 2019;69(3):e65-e69.

19. Clearfield HR. Diagnosing parietal abdominal pain. Hosp Pract (Off Ed). 1982;17(8):219,223,226-7.

20. Ecker AM, Donnellan NM, Shepherd JP, et al. Abdominal wall endometriosis: 12 years of experience at a large academic institution. Am J Obstet Gynecol. 2014;211(4):363.e1-363.e5.

21. Khan NAJ, Waseem S, Ullah S, et al. Slipping rib syndrome in a female adult with longstanding intractable upper abdominal pain. Case Rep Med. 2018;2018:7484560.

22. Whitcomb DC, Martin SP, Schoen RE, et al. Chronic abdominal pain caused by thoracic disc herniation. Am J Gastroenterol. 1995;90(5):835-7.

23. Brubaker ML, Sinaki M. Successful management of iliocostal impingement syndrome: a case series. Prosthet Orthot Int. 2016;40(3):384-7.

24. Siawash M, Mol F, Tjon A Ten W, et al. Anterior rectus sheath blocks in children with abdominal wall pain due to anterior cutaneous nerve entrapment syndrome: a prospective case series of 85 children. Paediatr Anaesth. 2017;27(5):545-50.

25. Van Assen T, de Jager-Kievit JWAJ, Scheltinga MR, et al. Chronic abdominal wall pain misdiagnosed as functional abdominal pain. J Am Board Fam Med. 2013;26(6):738-44.

26. Bishop WP. The Wall of Pain: Not All Abdominal Pain Is Visceral. J Pediatr Gastroenterol Nutr. 2016;62(3):359-60.
27. Scheltinga MR, Boelens OB, Tjon A Ten, et al. Surgery for refractory anterior cutaneous nerve entrapment syndrome (ACNES) in children. J Pediatr Surg. 2011;46(4):699-703.

28. Aranceta-Bartrina J, Gianzo-Citores M, Pérez-Rodrigo C. Prevalence of overweight, obesity and abdominal obesity in the Spanish population aged 3 to 24 years. The ENPE study. Rev Esp Cardiol (Engl Ed). 2020;73(4):290-9.

29. Bofill Moscardó I, Fernández Corchero A, Villegas Briz MA, et al. Psiquiatría infantil: patología prevalente en Atención Primaria, abordaje y tratamiento. Pediatr Aten Primaria 2010;12:s93-s106.

30. Boelens OB, Scheltinga MR, Houterman $S$, et al. Randomized clinical trial of trigger point infiltration with lidocaine to diagnose anterior cutaneous nerve entrapment syndrome. Br J Surg. 2013; 100:217-21.

31. Mol FMU, Jansen CH, Boelens OB, et al. Adding steroids to lidocaine in a therapeutic injection regimen for patients with abdominal pain due to anterior cutaneous nerve entrapment syndrome (ACNES): a single blinded randomized clinical trial. Scand J Pain. 2018;18(3):505-12.

32. Siawash M, Maatman R, Tjon A Ten W, et al. Anterior neurectomy in children with a recalcitrant anterior cutaneous nerve entrapment syndrome is safe and successful. J Pediatr Surg. 2017;52(3):47880.

33. Boelens OB, Van Assen T, Houterman $S$, et al. A double-blind, randomized, controlled trial on surgery for chronic abdominal pain due to anterior cutaneous nerve entrapment syndrome. Ann Surg. 2013;257(5):845-9.

34. Maatman RC, van Kuijk, Sander MJ, et al. A Randomized Controlled Trial to Evaluate the Effect of Pulsed Radiofrequency as a Treatment for Anterior Cutaneous Nerve Entrapment Syndrome in Comparison to Anterior Neurectomy. Pain Pract. 2019;19(7):75161. 